University of Wollongong

Research Online

Australian Institute for Innovative Materials -

Papers

Australian Institute for Innovative Materials

$1-1-2014$

Reinforcing biopolymer hydrogels with ionic-covalent entanglement hydrogel microspheres

Damian Martin Kirchmajer

University of Wollongong, dmk444@uowmail.edu.au

Marc in het Panhuis

University of Wollongong, panhuis@uow.edu.au

Follow this and additional works at: https://ro.uow.edu.au/aiimpapers

Part of the Engineering Commons, and the Physical Sciences and Mathematics Commons

Research Online is the open access institutional repository for the University of Wollongong. For further information contact the UOW Library: research-pubs@uow.edu.au 


\title{
Reinforcing biopolymer hydrogels with ionic-covalent entanglement hydrogel microspheres
}

\author{
Abstract \\ Microscopic hydrogel spheres can be used to improve the mechanical properties of conventional \\ hydrogels. We prepared ionic-covalent entanglement (ICE) hydrogel microspheres of calcium cross-linked \\ gellan gum and genipin cross-linked gelatin using a water-in-oil emulsion-based processing technique. \\ The method was optimized to produce microspheres with number average diameter $4 \pm 1 \mu \mathrm{m}$. These ICE \\ microspheres were used to reinforce gelatin hydrogels and improve their compressive mechanical \\ properties. The strongest microsphere reinforced hydrogels possessed a compressive mechanical stress \\ at failure of $0.50 \pm 0.1 \mathrm{MPa}$ and a compressive secant modulus of $0.18 \pm 0.02 \mathrm{MPa}$.

\section{Keywords} \\ biopolymer, hydrogels, ionic, reinforcing, covalent, microspheres, entanglement, hydrogel \\ Disciplines \\ Engineering | Physical Sciences and Mathematics

\section{Publication Details} \\ Kirchmajer, D. Martin. \& in het Panhuis, M. (2014). Reinforcing biopolymer hydrogels with ionic-covalent \\ entanglement hydrogel microspheres. Journal of Applied Polymer Science, 131 (15), 1-8.
}




\title{
Reinforcing biopolymer hydrogels with ionic-covalent entanglement hydrogel microspheres
}

Damian Martin Kirchmajer, Marc in het Panhuis

Soft Materials Group, School of Chemistry and Intelligent Polymer Research Institute, ARC Centre of Excellence for Electromaterials Science, AlIM Facility, University of Wollongong, Wollongong, NSW 2522, Australia

Correspondence to: Marc in het Panhuis (E-mail: panhuis@uow.edu.au)

((Additional Supporting Information may be found in the online version of this article.))

\begin{abstract}
Microscopic hydrogel spheres can be used to improve the mechanical properties of conventional hydrogels. We prepared ionic-covalent entanglement (ICE) hydrogel microspheres of calcium crosslinked gellan gum and genipin cross-linked gelatin using a water-in-oil emulsion based processing technique. The method was optimised to produce microspheres with number average diameter $4 \pm 1$ $\mu \mathrm{m}$. These ICE microspheres were used to reinforce gelatin hydrogels and improve their compressive mechanical properties. The strongest microsphere reinforced hydrogels possessed a compressive mechanical stress at failure of $0.50 \pm 0.1 \mathrm{MPa}$ and a compressive secant modulus of $0.18 \pm 0.02 \mathrm{MPa}$.

KEYWORDS ionic-covalent; gellan gum; gelatin; microspheres; reinforcement
\end{abstract}

\section{INTRODUCTION}

Tissue engineering technologies may one day provide a plentiful supply of highly compatible laboratory grown tissue and organs for implantation. ${ }^{1}$ The tissue scaffold, i.e. the material that reinforces the component's structure and holds the cells in place during maturation, is a critical component of all in vitro tissue engineering strategies. ${ }^{1}$ For engineering of soft tissues, hydrogel materials are the most appropriate scaffold materials because they have similar mechanical properties and chemical composition to natural tissues. ${ }^{2,3}$

Hydrogels are a class of polymeric materials whose composition comprises a small fraction of hydrophilic polymer network with water or aqueous solution as the major fraction. ${ }^{4}$ Hydrogels make excellent materials for engineered tissue scaffolds because they resemble the composition of natural extracellular matrix and possess mechanical properties similar to most soft tissue. ${ }^{5}$ Hydrogels are also excellent materials to use for drug delivery because they can easily absorb, retain and release bioactive molecules. ${ }^{6}$

Many hydrogels have very weak mechanical properties despite having excellent biocompatibility and utility as tissue scaffolds. Certain tissue types, such as cartilage, require scaffolds, which are strong, 
highly durable and resistant to fracture. Recently, topological hydrogels, ${ }^{7}$ tetra-PEG hydrogels, ${ }^{8}$ double network (DN) hydrogels, ${ }^{9-14}$ and ionic covalent entanglement (ICE) network hydrogels ${ }^{15-19}$ have been reported which are significantly stronger and tougher than conventional hydrogels. However, some of these strategies only work with very specific polymers and reagents and/or have very constricting methods for their preparation, which greatly limits the options for polymer choice and fabrication approach. For example, DN hydrogels must be prepared in a two-step method where one network is swollen in a monomer solution of the secondary network, a preparation method which is not amenable to printing or extrusion. ${ }^{10}$ Reinforcement of regular hydrogels with microgel particles is an alternative method for improving hydrogel properties which is in many ways more versatile than the abovementioned methods. ${ }^{20}$

Hydrogel microspheres can be used to reinforce hydrogels where they act as multifunctional crosslinking nodes. ${ }^{21,22}$ In a microgel reinforced hydrogel (MR-hydrogel), the microspheres inhibit the formation of micro-cracks and voids in the bulk hydrogel which are responsible for the rapid and catastrophic fracturing observed in unreinforced hydrogels. ${ }^{21}$ MR-hydrogels have also demonstrated effectiveness at presenting biological cues in a controlled fashion when used as a cartilage scaffold. ${ }^{23}$ Previously it was shown that double network hydrogel microspheres improved the mechanical properties of poly(acrylamide) hydrogels and we propose to use ICE hydrogel microspheres to strengthen gelatin hydrogels in a similar way. ${ }^{20,24,25}$ ICE hydrogels are an interpenetrating network of an ionotropic polymer and a chemically cross-linkable polymer which possess above average strength and toughness. ${ }^{15-18}$

A variety of methods have been reported for making hydrogel microspheres of gelatin and other polymers which have typically been used for drug delivery and controlled drug release applications. ${ }^{26-29}$ Of the many available options, emulsion based strategies offer a fast and scalable method for the production of nano- and micro- sized droplets which can be set as spheres upon cooling below the gel transition temperature. ${ }^{30}$ Basic water in oil emulsions are very easy to prepare and require only simple shearing that can, and often is, achieved with bench-top stirrers. However, these basic emulsions will prepare droplets/spheres between $10 \mu \mathrm{m}$ and $1000 \mu \mathrm{m}$ with a large size distribution. ${ }^{31}$ The finest emulsions are prepared under high shear and highly controlled shear in the presence of an appropriate emulgent where almost monodisperse droplets form as a result of Rayleigh instability. ${ }^{31}$ Many factors affect the ability to emulsify a solution (for example, of gellan gum and gelatin), including the choice of emulgents, emulgent concentration, and the choice of oil. ${ }^{31}$

The microspheres presented in this manuscript are comprised of an ICE hydrogel prepared from calcium cross-linked gellan gum and genipin cross-linked gelatin. Gellan gum is a polysaccharide biopolymer produced from the bacteria Pseudomonas elodea that forms a firm gel upon cooling to below its gel transition temperature $\left(\sim 45^{\circ} \mathrm{C}\right)^{32-34}$. The strength of gellan gum hydrogels is significantly affected by the presence of cross-linking cations and in particular, calcium cations produce strong thermally irreversible hydrogels that enable microspheres to be produced from emulsions by thermal modulation. ${ }^{35,36}$ Gelatin is a proteinaceous biopolymer produced from the controlled hydrolysis of collagen. ${ }^{37}$ Ordinarily, gelatin hydrogels swell extensively; rapidly dissolve above the gel transition temperature $\left(\sim 29^{\circ} \mathrm{C}\right)$, and lose their strength and rigidity rapidly. ${ }^{37-39}$ However, covalently cross-linking the gelatin with genipin (the algycone 
of geniposide, an iridoid glycoside that is the major component of the fruit of the gardenia plant, Gardenia jasmindides Ellis) ${ }^{40}$ remedies these limitations. ${ }^{41,42}$ Genipin forms chemical cross-links mainly between the primary amino groups of either the $\varepsilon$-amino groups of the lysine residues in gelatin and it is this reaction that is responsible for both the formation of the ICE network but also the coupling of the microsphere phases to the bulk hydrogel phase in MR-hydrogels. ${ }^{43}$

In this paper, we present a simple method for the preparation of gellan gum-gelatin ICE hydrogel microspheres. The optimal emulgent hydrophile-lipophile balance (HLB) was identified, and the effect of emulgent concentration was explored. A variety of different oils were examined for their effectiveness at producing the microsphere-forming emulsions. The size of the resulting microspheres was characterised and their ability to reinforce gelatin hydrogels was evaluated.

\section{EXPERIMENTAL}

\section{Materials and equipment}

Hydrogels were prepared from acid hydrolysed porcine gelatin (Bloom number of 300, molecular weight of $87,500 \mathrm{Da}$, Sigma Aldrich, USA) and low acyl gellan gum (CP Kelco, Singapore) using deionised water (DI) which had been purified using a combination ion-exchange/reverse osmosis filtration system (Millipore, USA) to a resistivity of $18 \mathrm{M} \Omega \mathrm{cm}$. The hydrogels were cross-linked using solutions of genipin (Challenge Bioproducts, Taiwan) and $\mathrm{CaCl}_{2}$ (Sigma Aldrich, USA). Water-in-oil (w/o) emulsions were formed using canola oil (Crisco, USA), hydraulic oil (Ultramax 46, Valvoline), machine oil (X68, Caltex), 3in-1 oil (3-in-1, USA) and paraffin oil (Recochem, Australia) with non-ionic surfactants Tween 80 (Sigma Aldrich, USA) and Span 80 (Merck, Australia). Solvents used for washing residual oils and surfactants from hydrogel microspheres included toluene (BDH, Australia), petroleum spirit (BDH, Australia), dichloromethane (Ajax Finechem, Australia), acetone (Ajax Finechem, Australia) and ethyl acetate (BDH, Australia).

\section{Emulgent preparation}

Emulgent mixes of different HLB values were prepared by combining Tween 80 and Span 80 to different proportion by weight. HLB values of the emulgent mixture $(X)$ were calculated using:

$X=f_{\text {Tween } 80} H L B_{\text {Tween } 80}+f_{\text {Tween } 80} H L B_{\text {Tween } 80}$,

where $f_{\text {Tween } 80}$ and $f_{\text {Span } 80}$ are the weight fractions of each surfactant; $H L B_{\text {Span } 80}$ and $H L B_{\text {Tween } 80}$ are the HLB values for each surfactant (4.3 and 15.0, respectively); and $X$ is the desired HLB of the emulgent mixture. Table 1 provides a reference chart describing the exact ratios of Tween 80 and Span 80 used to prepare the different emulgent mixtures used in this research and their corresponding HLB values.

TABLE 1

\begin{tabular}{llllllllll}
\hline Tween $80\left(\% w w^{-1}\right)$ & 100 & 90 & 80 & 70 & 60 & 50 & 40 & 30 & 20 \\
\hline
\end{tabular}




\begin{tabular}{cccccccccc}
\hline Span 80 (\%w w $\left.\mathbf{w}^{-1}\right)$ & 0 & 10 & 20 & 30 & 40 & 50 & 60 & 70 & 80 \\
HLB & 15 & 13.93 & 12.86 & 11.79 & 10.72 & 9.65 & 8.58 & 7.51 & 6.44 \\
\hline
\end{tabular}

\section{Microsphere fabrication}

ICE hydrogel solutions comprising $1 \%\left(w^{-1}\right)$ gellan gum, $1.75 \%\left(w v^{-1}\right)$ gelatin and $2 \%\left(w w^{-1}\right) \mathrm{Ca}^{2+}$ were prepared by dissolving the gellan gum and gelatin in DI water at $80^{\circ} \mathrm{C}$ with gentle stirring on a combined magnetic stirrer/hot plate (CB162, Stuart, UK) for 30 minutes before $1 \mathrm{M} \mathrm{CaCl}_{2}$ solution was added. Oil/emulgent mixtures were prepared separately at $60^{\circ} \mathrm{C}$. Unless otherwise stated, the oil/emulgent mixtures were $0.1 \%\left(w w^{-1}\right)$ Tween 80 and $0.9 \%\left(w w^{-1}\right)$ Span 80 in paraffin oil.

Emulsions, unless otherwise stated, were prepared using a tissue homogeniser (HG-15D Wisemix, Daihan Scientific, Korea) with dispersing tool (HT1018, Wisemix, Daihan Scientific, Korea) which was prewarmed to $60^{\circ} \mathrm{C}$ by immersing the tool in hot water. $200 \mathrm{~mL}$ of hydrogel solution was added slowly to $300 \mathrm{~mL}$ of oil-emulgent mixture whilst stirring at 2,000 rpm in a $600 \mathrm{~mL}$ low-form glass beaker (Crown, Australia). After the solutions were combined, the speed was increased to 7,000 rpm and maintained for 5 minutes (Figure 1i). The emulsion was kept warm throughout the homogenising using a hotplate (CB162, Stuart, UK).

The emulsion was gelled into a microsphere suspension immediately after stirring by pouring it into an ice-cold, $1 \mathrm{~L}$ glass bottle (Schott, Germany), and then plunging the bottle into a bucket of ice for approximately 15 minutes (Figure 1ii). The oil and emulgents were removed from the microspheres by washing with two $500 \mathrm{~mL}$ portions of petroleum spirits, and one $450 \mathrm{~mL}$ portion of petroleum spirits with $50 \mathrm{~mL}$ ethyl acetate added (Figure 1iii). The microspheres were then freeze dried completely (Alpha LD plus, Christ, Germany) (Figure 1iv).

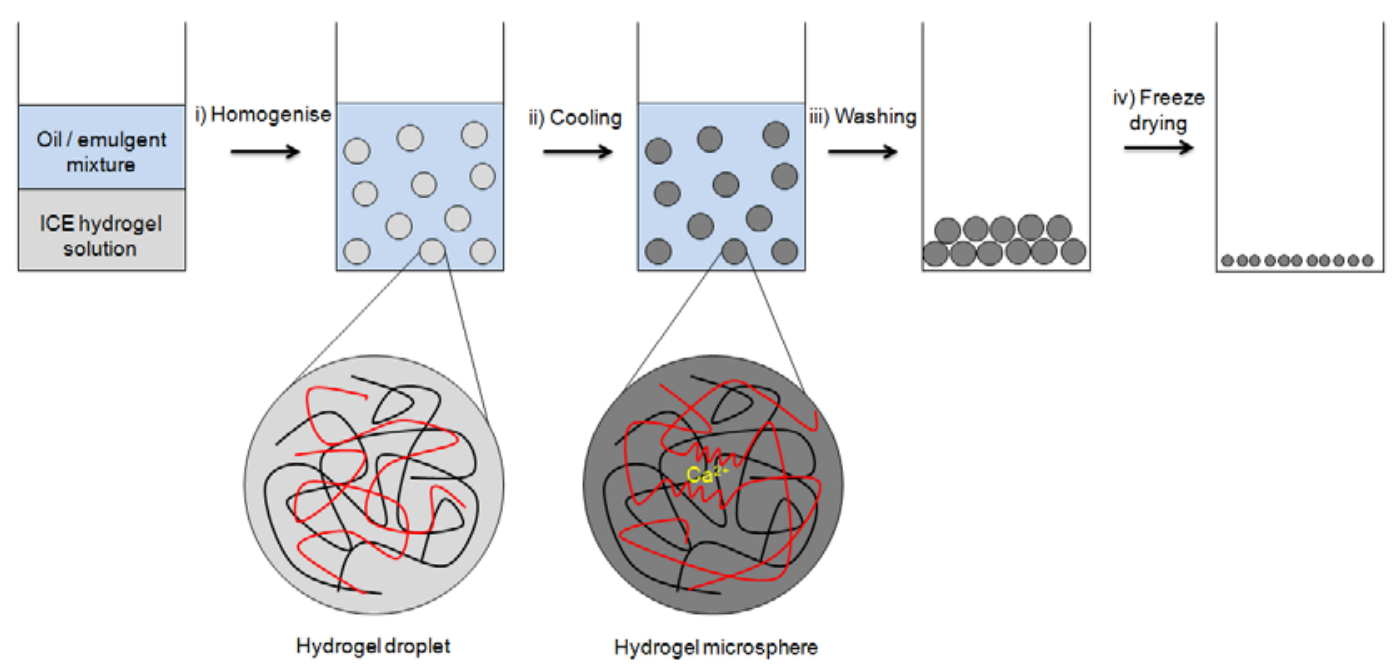


Figure 1 Schematic representation of the procedure for the fabrication of ICE hydrogel microspheres using emulsification to create micro-sized droplets of hydrogel solution and thermal modulation to set the droplets into firm hydrogel microspheres

\section{MR-hydrogel preparation}

MR-hydrogels were prepared by combining freeze-dried microspheres with a solution of gelatin at $50^{\circ} \mathrm{C}$ and stirring gently for 10 minutes to allow the microspheres to swell and rehydrate (Figure 2i). Subsequently, the solution was cross-linked by addition of genipin solution ( $20.3 \% \mathrm{w} / \mathrm{w}$ in $60 \%$ ethanol) to a concentration of $200 \mathrm{mg}$ genipin per gram gelatin and stirring for a further three minutes (Figure 2ii). The gel solution was then poured into glass Petri dish moulds (60 mm diameter x $15 \mathrm{~mm}$ height, Schott, Australia) and left to cure, covered, for 24 hours at $21^{\circ} \mathrm{C}$. Silanisation of the Petri dishes using Coatasil (Ajax Finechem, Australia) glass treatment solution prior to casting facilitated easy removal of the cured hydrogels from the dishes. The swelling ratio $(S W)$ of hydrogels were calculated as the mass of the swollen hydrogel $\left(m_{s}\right)$ divided by the mass of the dried hydrogel $\left(m_{d}\right)$,

$S W=m_{s} / m_{d}$

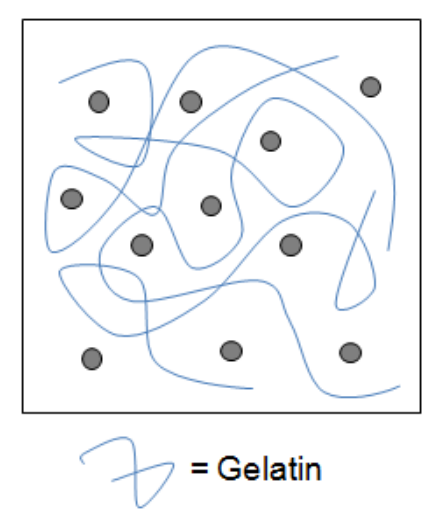

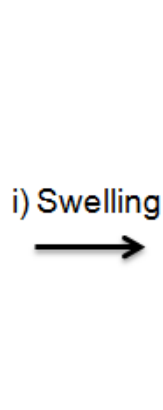

(2)

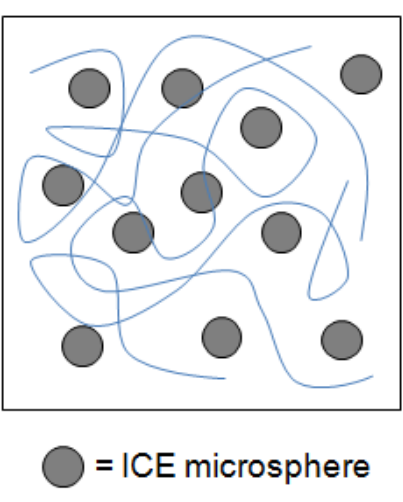

(2)

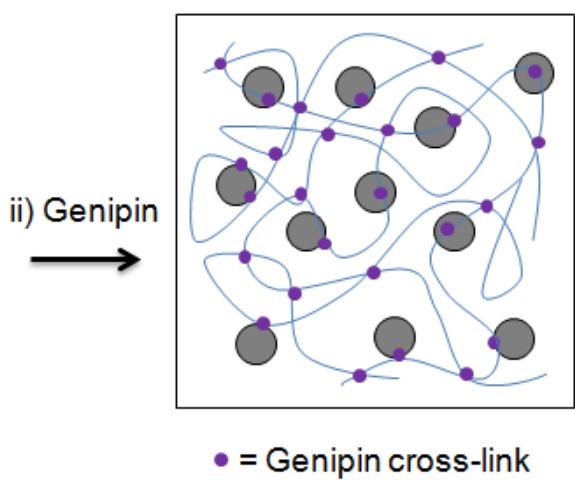

Figure 2: Schematic representation of the procedure for preparation of MR-hydrogels.

\section{Rheology}

The viscosities of the gel solution and various oils were analysed using a rheometer (Physica MCR-301, Anton Paar, Australia) with temperature controlled stage (AWC100, Julabo, Germany), a $50 \mathrm{~mm}$ diameter and $1^{\circ}$ cone and plate tool (CP50-1, Anton Paar, Australia), with a gap length of $0.097 \mathrm{~mm}$. The viscosity was measured over a range of temperatures spanning from $80^{\circ} \mathrm{C}$ down to $4^{\circ} \mathrm{C}$ by applying a rotational shear of $100 \mathrm{~s}^{-1}$ while gradually lowering the temperature at a rate of $-5^{\circ} \mathrm{C} \mathrm{min}^{-1}$.

\section{Size analysis}

An optical microscope (DM6000, Leica, Germany) was used to take micrographs of the microspheres. Leica Application Suite (Leica, Germany) was used to measure the size and size distribution of the microspheres. Samples prepared for optical microscopy were prepared by suspending the microspheres 
in DI water and dropping onto glass microscopy slides. Freeze dried microspheres were rehydrated for 10 minutes in DI water prior to analysis.

\section{Mechanical analysis}

The mechanical properties of the hydrogels were examined in compression using a universal analyser (EZ-S, Shimadzu, Japan). Samples were cut from slabs of hydrogel into rectangular prisms $10 \mathrm{~mm} \times 10$ $\mathrm{mm} \times 7 \mathrm{~mm}$, and subsequently compressed at a rate of $2 \mathrm{~mm} \mathrm{~min}^{-1}$ at $21^{\circ} \mathrm{C}$. The resulting stress-strain data was used to determine the compressive failure strain $\left(\varepsilon_{c}\right)$, compressive secant modulus over $20 \%$ $30 \%$ strain $\left(E_{c}\right)$, compressive failure stress $\left(\sigma_{c}\right)$ and compressive strain energy to failure $(U)$.

\section{RESULTS AND DISCUSSION}

\section{Optimisation of microsphere production}

Microspheres of ICE hydrogels were prepared using emulsion based processing and thermal modulation of the gel solution (Figures 1 and 2). It was found that ICE hydrogel microspheres could be prepared in approximately 1-2 hours and that it could be performed easily on a $200 \mathrm{~mL}$ scale which is much quicker and larger scale than all previously reported DN microspheres which took over 8 hours to prepare a significantly smaller quantity. ${ }^{20,24,25}$ The optimal process for preparing hydrogel microspheres was determined by independently examining the effects of i) the type and viscosity of oil; ii) the emulgent HLB value; and iii) the emulgent concentration.

The hydrophile-lipophile balance (HLB) of the emulgent used to prepare emulsions was observed to have an effect on the type of emulsion formed, its stability, and the ultimate quality of the microspheres produced. Emulgents with HLB values between 4.3 and 15 were used to prepare emulsions of paraffin oil and gel solution. The emulsions were assessed qualitatively by inspecting micrographs of the emulsions, which had to be set by dropping the temperature immediately after shearing ceased (Figure 3). The stability of the emulsions was also assessed qualitatively by comparing micrographs of the same emulsion taken immediately after shearing ceased and 10 minutes afterwards. Emulsion prepared with emulgent HLB values between 15.0 and 11.8 formed oil-in-water (o/w) type emulsions. These emulsions created porous hydrogel sponges with droplets of oil trapped in the pores after being cooling. Emulsions prepared with HLB values of 10.7 and 9.6 produced oil-in-water-in-oil (o/w/o) double emulsions initially, which would transform into single o/w emulsions gradually over 10 minutes. Emulsions prepared with emulgent HLB values of 8.6 and 7.5 produced unstable water-in-oil (w/o) emulsions that would coalesce and agglomerate noticeably over 10 minutes. The best quality hydrogel microspheres and most stable w/o emulsions were prepared using emulgents with HLB values between 4.5 and 6.4. 


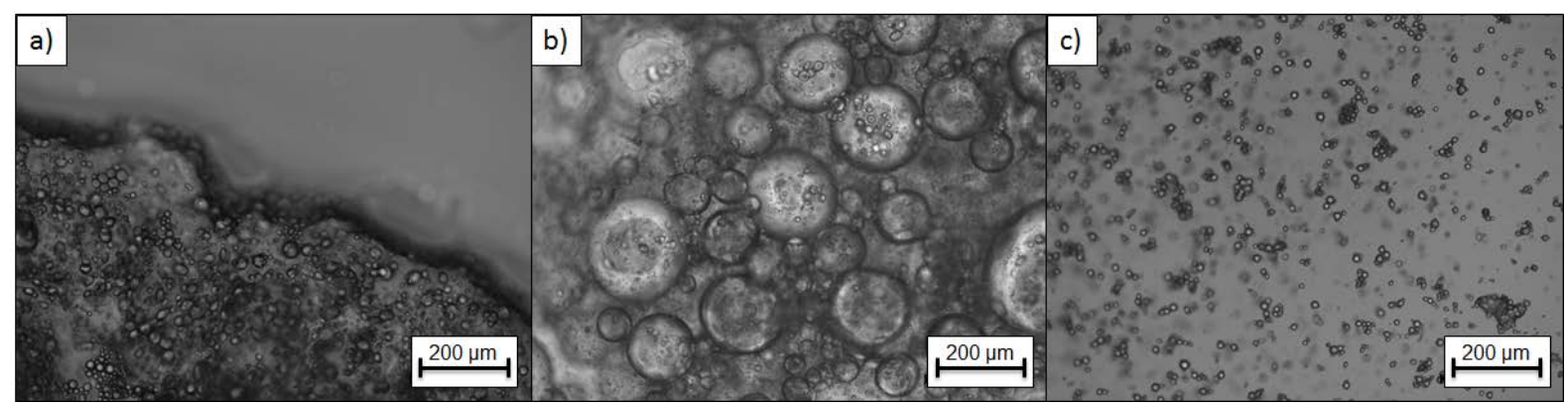

Figure 3 Micrographs (taken immediately after shearing ceased) of emulsions prepared with different HLB values. a) An o/w emulsion formed with emulgent HLB value of 13.9. b) An o/w/o emulsion formed with emulgent HLB value of 9.6. c) A w/o emulsion formed with emulgent HLB value of 6.4.

The concentration of emulgent affected the stability of the emulsion and the size of microspheres prepared from the emulsion. Emulsions were prepared using different concentrations of emulgent (HLB value $=5.4)$ ranging from $0.1 \%(w / w)$ to $8 \%(w / w)$. Emulsions formed with $0.1 \%$ emulgent were not stable and coalesced back into two separate layers before the gel solution set. When $0.5 \%$ or more emulgent was used, stable emulsions were formed for long enough to cool the gel solution to below the gel transition point and form microspheres. With increasing emulgent concentration, the average microsphere diameter decreased as did the size distribution of the microsphere (Figure 4a). When more than $4 \%$ emulgent was used the microspheres produced were very small $(<10 \mu \mathrm{m})$ and highly stabilised in paraffin oil by the presence of a large amount of surfactant; this made it difficult to separate the microspheres from the oil after setting. In general, the time it took for microspheres to settle out of suspension increased in proportion to the concentration of emulgent used and was between 5 minutes and 2 hours for all concentrations tested excepting those made with $8 \%\left(w w^{-1}\right)$ emulgent which took one week to settle (Figure $4 \mathrm{~b}$ ). An optimal range of emulgent concentration was identified as $1-2 \%$ ( $w$ $\left.\mathrm{w}^{-1}\right)$, where the average diameter and size distribution of microspheres are minimised and the ease of processing them was maximised. 
a)

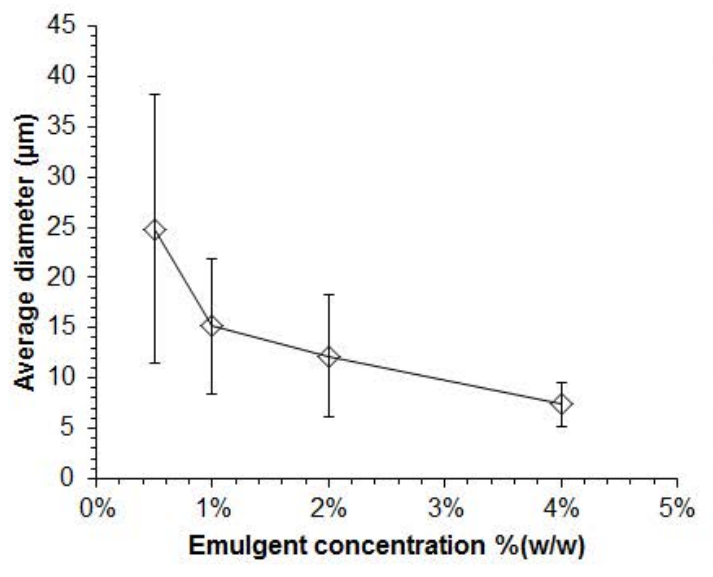

b)

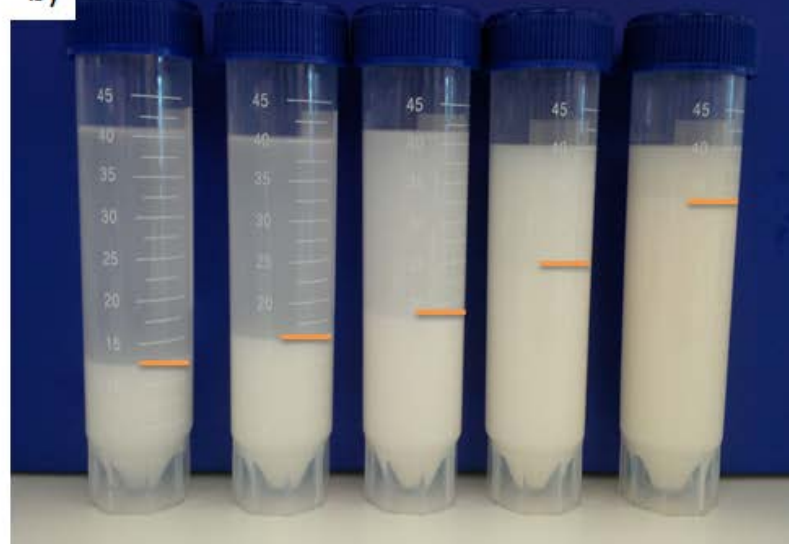

Figure 4 a) The number average diameter of microspheres prepared from emulsions with different concentration of emulgent. Error bars indicate the standard distribution of microsphere diameters which also decrease with increasing emulgent concentration. b) Photograph of $10 \mathrm{~mL}$ of microspheres taken approximately 5 minutes after resuspending in $30 \mathrm{~mL}$ of petroleum spirit. Orange lines on the photo indicate the extent of settling at this point in time - the settling time increases with increasing emulgent used to prepared the microspheres. The concentration of emulgent used to prepare the microspheres increases from left to right in sequence: $0.5 \%, 1.0 \%, 2.0 \%, 4.0 \%, 8.0 \%$.

Rheological measurements of ICE hydrogel solution over the temperature range of $80-10^{\circ} \mathrm{C}$ indicated that the gel transition temperature of the ICE network was approximately $45^{\circ} \mathrm{C}$ (Figure 5a). The viscosities of five different oils were also examined over the same temperature range and found to range from 11 to $42 \mathrm{mPa} s$ at a shear rate of $100 \mathrm{~s}^{-1}$ at $45^{\circ} \mathrm{C}$ (Figure 5b). These five different oils were then used to prepare ICE microspheres under identical conditions (1\% emulgent with HLB value of 5.4) and it was observed that the type of oil affected the size of the microspheres produced which ranged from $14-60 \mu \mathrm{m}$ and with varying size distributions (Table 2). A correlation between the size distribution (expressed as the relative standard deviation of the diameter measurements) of the microspheres and the viscosity of the oil used to prepare them was observed (Figure 5b) - in general, it was observed that higher viscosity oils, produced microspheres with smaller size distributions. This observation is explicable as the effect caused Rayleigh instability which causes droplets in an emulsion to fragment towards a size distribution that is dependent on the relative viscosities of the dispersed and continuous phases. ${ }^{31}$ Despite the large size distribution attained through using paraffin oil (the lowest viscosity oil), it was selected for continued use throughout our experiments because it was the easiest oil to extricate fully from the microspheres after fabrication. 

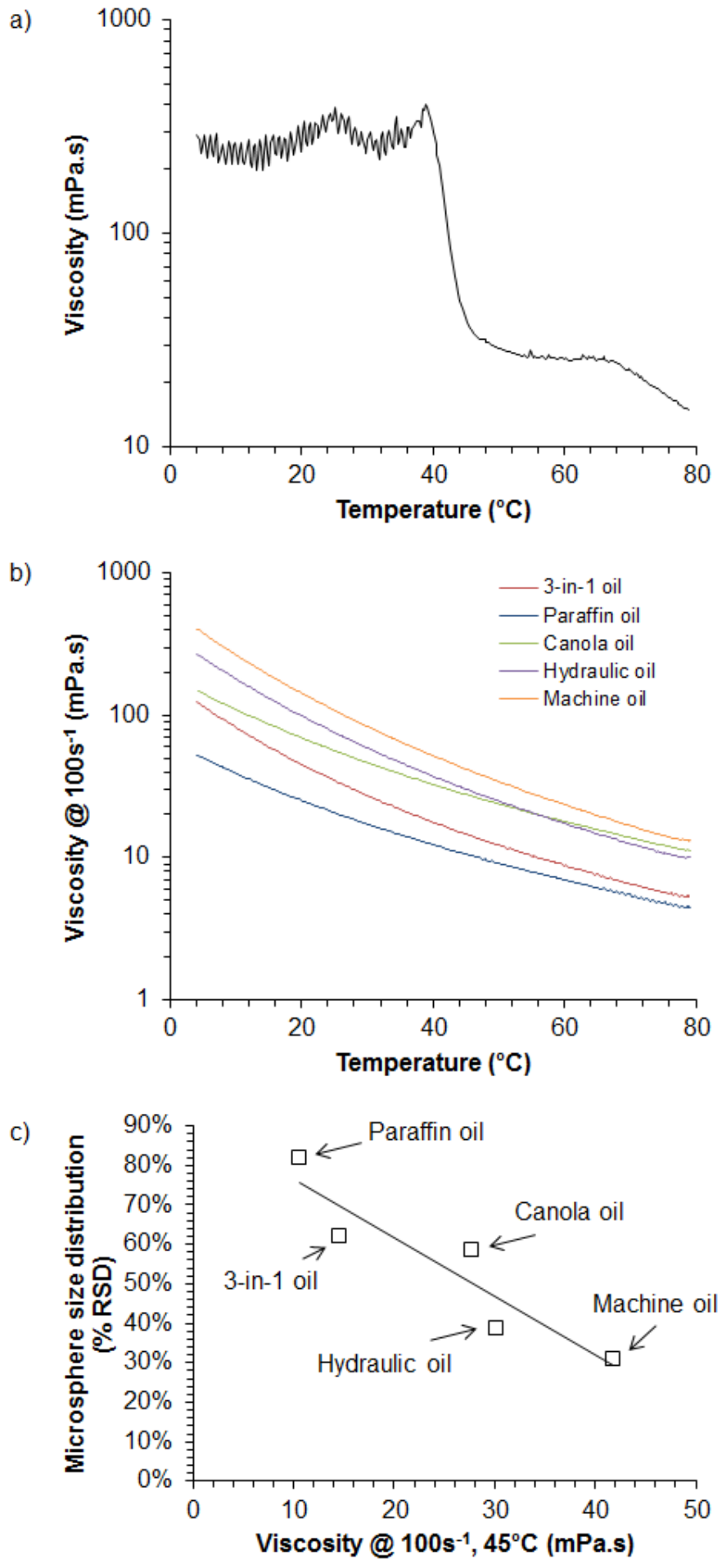

Figure 5 a) Viscosity at shear rate $100 \mathrm{~s}^{-1}$ versus temperature during cooling from $80^{\circ} \mathrm{C}$ to $10{ }^{\circ} \mathrm{C}$ of ICE hydrogel solution, b) viscosity at shear rate $100 \mathrm{~s}^{-1}$ versus temperature of different oils $\mathrm{c}$ ) Microsphere size distribution expressed as relative standard deviation versus oil viscosity (at shear rate $100 \mathrm{~s}^{-1}$, temperature $45^{\circ} \mathrm{C}$ ). Solid line is a guide to the reader's eye. 
Table 2 Viscosity at shear rate $100 \mathrm{~s}^{-1}$ and temperature $45^{\circ} \mathrm{C}(\eta)$ for different oil types used to prepare ICE microspheres and their corresponding microsphere number average diameter (NAD) values.

\begin{tabular}{lll}
\hline Oil type & $\begin{array}{l}\eta \\
(\mathrm{mPa})\end{array}$ & $\begin{array}{l}\text { NAD } \\
(\mu \mathrm{m})\end{array}$ \\
\hline Paraffin & $11 \pm 1$ & $60 \pm 50$ \\
3-in-1 & $15 \pm 2$ & $14 \pm 9$ \\
Canola & $28 \pm 2$ & $14 \pm 8$ \\
Hydraulic & $30 \pm 2$ & $50 \pm 20$ \\
Machine & $42 \pm 3$ & $25 \pm 8$ \\
\hline
\end{tabular}

\section{Mechanical reinforcement of hydrogels}

Gellan gum-gelatin ICE hydrogel microspheres were used to reinforce gelatin hydrogels (Figure 6a). These hydrogels were prepared with a total polymer concentration of $6 \%(w / w)$ (swelling ratio, SW $=18$ \pm 1 ) made up of gelatin and freeze-dried ICE microspheres in different proportions. The freeze-dried microspheres were observed to hydrate and swell almost immediately upon mixing with the polymer solution to a final size of $4 \pm 1 \mu \mathrm{m}$ (Figure $6 \mathrm{~b}$ ). 

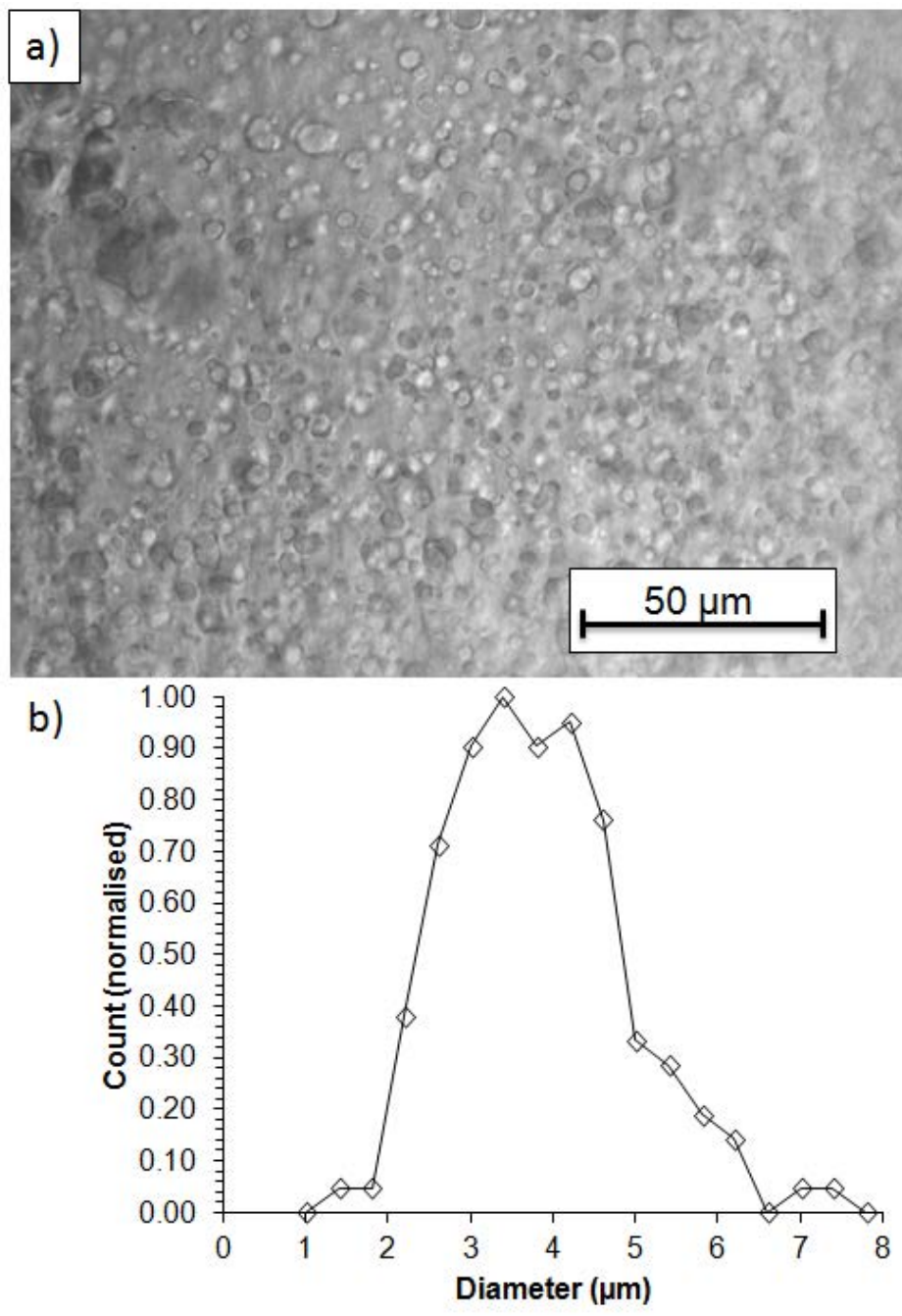

Figure 6 a) Micrograph of gelatin hydrogel which has been reinforced by replacing $40 \%$ of the polymer with freeze-dried microspheres; b) size distribution of microspheres in MR-hydrogels.

Hydrogels comprised entirely of genipin cross-linked gelatin (no added microspheres) were relatively soft and ductile and possessed compressive failure stress of $0.41 \pm 0.09 \mathrm{MPa}$, compressive strain to failure of $69 \pm 2 \%$, compressive secant modulus of $0.074 \pm 0.008 \mathrm{MPa}$ and compressive strain energy to failure of $39 \pm 7 \mathrm{~kJ} \mathrm{~m}^{-3}$. The MR-hydrogels exhibited a maximum in mechanical properties at a microsphere fraction of $40 \%$ (Figure 7). In comparison to gelatin only hydrogels, the MR-hydrogels were stiffened (increase in $E_{c}$ to $0.18 \pm 0.02 \mathrm{MPa}$ ) without losing their ductility and had a resultant increase in compressive strain energy to failure to $70 \pm 10 \mathrm{~kJ} \mathrm{~m}^{-3}$.

In comparison to DN MR-hydrogels presented in recent literature our hydrogels appear to be weaker, which can (partially) be attributed to the difference in swelling ratio. The PAMPS/PAAm microgel reinforced PAAm hydrogels reported by Gong et al. possessed tensile fracture stresses of $2.46 \mathrm{MPa}$ and Young's modulus of $0.22 \mathrm{MPa}$, however, these hydrogels had a low swelling ratio of $6.7 .^{24}$ The scale that 
our ICE MR-hydrogels were prepared was also significantly larger ( $15 \mathrm{~mL}$ pieces of hydrogel) than the DN MR-hydrogels ( $1 \mathrm{~mL}){ }^{24}$

Increasing the microsphere fraction above $40 \%$ led to a reduction in the values of the mechanical properties (Figure 7). For example, increasing the fraction from $40 \%$ to $60 \%$ resulted in a significant reduction in stress at failure (from 0.5 $\mathrm{MPa}$ down to $0.1 \mathrm{MPa}$ ). When gels were composed entirely of microspheres (100\%), a self-supporting but weak gel, which behaved like a semi-viscous fluid with an undetermined yield stress was apparent. This indicates that ratio of microspheres to bulk phase polymer is an important determinant of the mechanical performance of these hydrogels.

The mechanical behaviour of our gels is consistent with MR-hydrogels being a two-phase composite consisting of gelatin network and microgels. ${ }^{24}$ It has been previously proposed that the stiffening (increase in $E_{c}$ ) is a result from topologically constrained chain entanglements of the gelatin matrix and the ICE microgels. ${ }^{24}$

a)

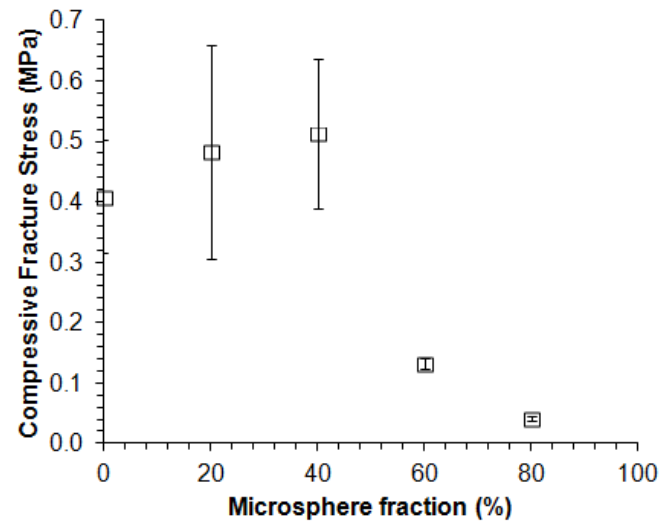

c)

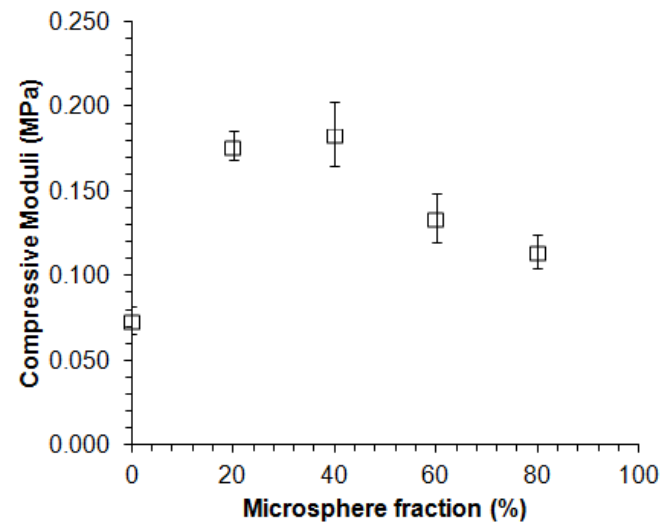

b)

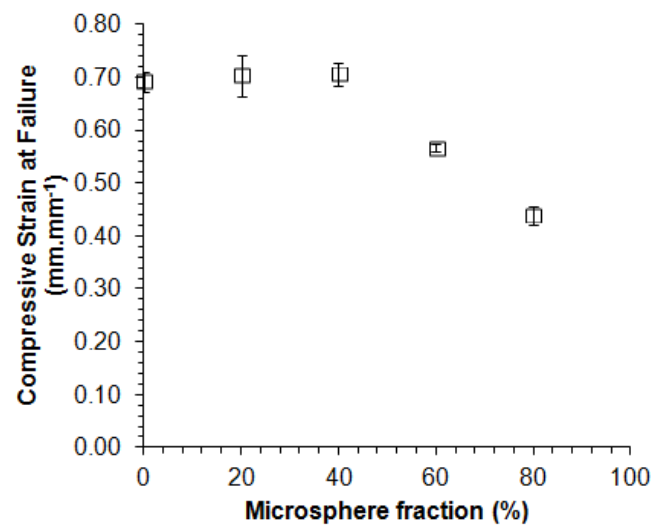

d)

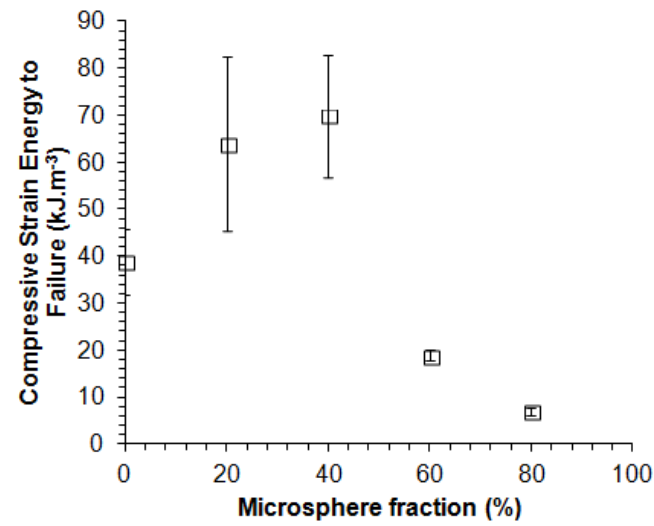

Figure 7 Compressive mechanical properties of $6 \%$ gelatin hydrogels where $0 \%$ to $80 \%$ of the polymer is replaced by gellan gum-gelatin ICE hydrogel microspheres. 


\section{CONCLUSIONS}

The preparation of a new type of microspheres based on gellan gum-gelatin ionic-covalent entanglement hydrogels using the oil-in-water emulsion method is reported. It was determined that the optimal mixture of emulgents was 1 part Tween 80 to 9 parts Span 80 (by weight) which produced an emulgent mixture with a HLB value of 5.4. It was observed that the emulgent concentration was inversely proportionate to microsphere number average diameter. However, the optimal concentration of emulgent was considered to be $1 \%\left(w w^{-1}\right)$ as it provided a good compromise between small microsphere diameter and ease of process-ability. A variety of different oils were also successfully used to prepare the microspheres and a correlation between oil viscosity and size distribution was observed.

Microspheres were used to mechanically reinforce gelatin hydrogels where it was demonstrated that an optimal proportion of microspheres was $40 \%(w / w)$ of the total polymer in the hydrogel. This optimized proportion produced hydrogels with swelling ratio $18 \pm 1$, which exhibited reinforcement compared to the gelatin matrix.

\section{ACKNOWLEDGEMENTS}

This work was funded by the University of Wollongong and the Australian Research Council Centre of Excellence and Future Fellowship programs. The authors acknowledge use of facilities within the UOW Electron Microscopy Centre.

\section{REFERENCES AND NOTES}

1. Langer, R.; Vacanti, J. P. Science 1993, 260, 920-926.

2. Ma, P. X. Adv. Drug Deliv. Rev. 2008, 60, 184-198.

3. Lee, K. Y.; Mooney, D. J. Chem. Rev. 2001, 101, 1869-1879.

4. Calvert, P. Adv. Mater. 2009, 21, 743-756.

5. Drury, J. L.; Mooney, D. J. Biomaterials 2003, 24, 4337-4351.

6. Vasheghani-farahani, E.; Ganji, F. Polym. J. 2009, 18, 63-88.

7. Okumura, Y.; Ito, K. Adv. Mater. 2001, 8656, 485-487.

8. Shibayama, M. Soft Matter 2012, 8, 8030-8038.

9. Gong, J. P.; Katsuyama, Y.; Kurokawa, T.; Osada, Y. Adv. Mater. 2003, 15, 1155-1158.

10. Gong, J. P. Soft Matter 2010, 6, 2583-2590.

11. Nakayama, A.; Kakugo, A.; Gong, J. P.; Osada, Y.; Takai, M.; Erata, T.; Kawano, S. Adv. Funct. Mater. 2004, 14, 1124-1128.

12. Webber, R. E.; Creton, C.; Brown, H. R.; Gong, J. P. Macromolecules 2007, 40, 2919-2927.

13. Suekama, T. C.; Hu, J.; Kurokawa, T.; Gong, J. P.; Gehrke, S. H. Macromol. Symp. 2013, 329, 9-18.

14. Hu, J.; Kurokawa, T.; Nakajima, T.; Sun, T. L.; Suekama, T.; Wu, Z. L.; Liang, S. M.; Gong, J. P. Macromolecules 2012, 45, 9445- 9451. 
15. Stevens, L.; Calvert, P.; Wallace, G. G.; in het Panhuis, M. Soft Matter 2013, 9, 3009-3012.

16. Bakarich, S. E.; Pidcock, G. C.; Balding, P.; Stevens, L.; Calvert, P.; in het Panhuis, M. Soft Matter 2012, 8, 9985-9988.

17. Sun, J.-Y.; Zhao, X.; Illeperuma, W. R. K.; Chaudhuri, O.; Oh, K. H.; Mooney, D. J.; Vlassak, J. J.; Suo, Z. Nature 2012, 489, 133-136.

18. Bakarich, S. E.; in het Panhuis, M.; Beirne, S.; Wallace, G. G.; Spinks, G. M. J. Mater. Chem. B 2013, 1, 4939.

19. Chen, Q.; Zhu, L.; Zhao, C.; Wang, Q.; Zheng, J. Adv. Mater. 2013, 25, 4171-4176.

20. Lane, T.; Holloway, J. L.; Milani, A. H.; Saunders, J. M.; Freemont, A. J.; Saunders, B. R. Soft Matter 2013, 9, 7934.

21. Huang, T.; Xu, H.; Jiao, K.; Zhu, L.; Brown, H. R.; Wang, H. Adv. Mater. 2007, 19, 1622-1626.

22. Qin, X.; Zhao, F.; Liu, Y.; Wang, H.; Feng, S. Colloid Polym. Sci. 2009, 287, 621-625.

23. Jha, A. K.; Malik, M. S.; Farach-carson, M. C.; Duncan, L.; Jia, X. Soft Matter 2010, 6, 5045-5055.

24. Hu, J.; Hiwatashi, K.; Kurokawa, T.; Liang, S. M.; Wu, Z. L.; Gong, J. P. Macromolecules 2011, 44, 7775-7781.

25. Hu, J.; Kurokawa, T.; Hiwatashi, K.; Nakajima, T.; Wu, Z. L.; Liang, S. M.; Gong, J. P. Macromolecules 2012, 45, 5218-5228.

26. Ethirajan, A.; Schoeller, K.; Musyanovych, A.; Ziener, U.; Landfester, K. Biomacromolecules 2008, 9, 2383-2389.

27. Young, S.; Wong, M.; Tabata, Y.; Mikos, A. G. J. Control. Release 2005, 109, 256-74.

28. Thakur, G.; Mitra, A.; Rousseau, D.; Basak, A.; Sarkar, S.; Pal, K. J. Mater. Sci. Mater. Med. 2011, 22, 115-123.

29. Liang, H. C.; Chang, W. H.; Lin, K. J.; Sung, H. W. J. Biomed. Mater. Res. 2003, 65, 271-282.

30. Landfester, K.; Musyanovych, A. Adv. Polym. Sci. 2010, 234, 39-63.

31. Leal-Calderon, F.; Veronique, S.; Bibette, J. Emulsion Science: Basic Principles; Springer: New York, 2007.

32. Smith, A. M.; Shelton, R. M.; Perrie, Y.; Harris, J. J. J. Biomater. Appl. 2007, 22, 241-254.

33. Jansson, P.-E.; Lindberg, B. Carbohydr. Res. 1983, 124, 135-139.

34. O'Neill, M. A.; Selvendran, R. R.; Morris, V. J. Carbohydr. Res. 1983, 124, 123-133.

35. Morris, E. R.; Gothard, M. G. E.; Hember, M. W. N.; Manning, C. E.; Robinson, G. Carbohydr. Polym. 1996, 30, 165-175.

36. CP Kelco. Product Infromtation: KELCOGEL ${ }^{\circledR}$ Gellan Gum; 2007.

37. Djabourov, M.; Leblond, J.; Papon, P. J. Phys. 1988, 49, 319-332.

38. Djabourov, M.; Leblond, J.; Papon, P. J. Phys. 1988, 49, 333-343.

39. Ross-Murphy, S. B. Polymer 1992, 33, 2622-2627.

40. Slusarewicz, P.; Zhu, K.; Hedman, T. Nat. Prod. Commun. 2010, 5, 1853-1858.

41. Bigi, A.; Cojazzi, G.; Panzavolta, S.; Roveri, N.; Rubini, K. Stabilization of Gelatin Films by Crosslinking with Genipin. Biomaterials 2002, 23, 4827-4832.

42. Kirchmajer, D. M.; Watson, C. A.; Ranson, M.; in het Panhuis, M. RSC Adv. 2013, 3, 1073-1081.

43. Nickerson, M. T.; Patel, J.; Heyd, D. V; Rousseau, D.; Paulson, A. T. Int. J. Biol. Macromol. 2006, 39, 298-302. 



\section{GRAPHICAL ABSTRACT}

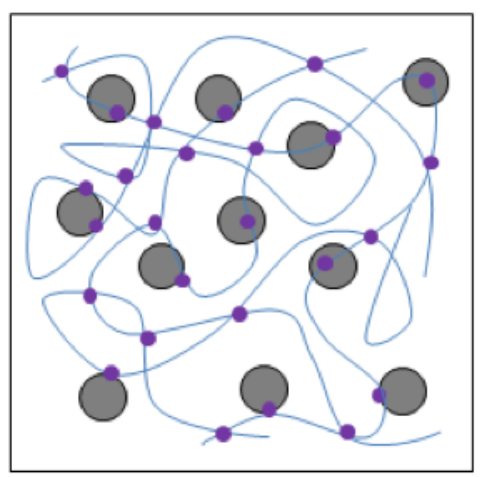

